\title{
Refining the Phase I pediatric trial
}
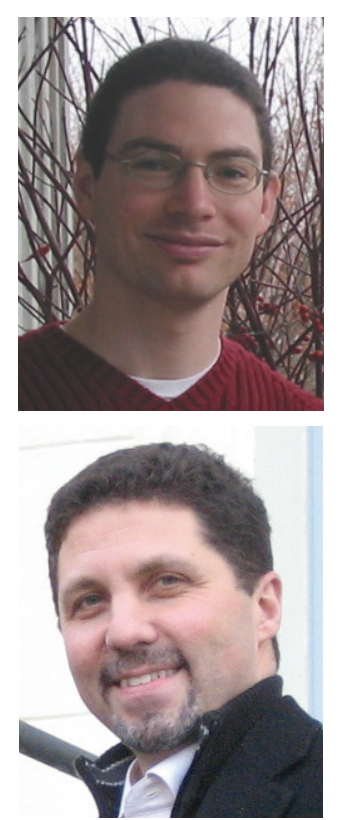

Jeffrey M Skolnik ${ }^{1,2 \dagger}$ \& Jeffrey S Barrett ${ }^{2}$

${ }^{\dagger}$ Author for correspondence ${ }^{1}$ AstraZeneca, LP, Mail Stop C2B-112, 1800 Concord Pike, PO Box 15437, Wilmington, DE 19850-5437, USA Tel.: + 13028857686 ; Fax: +1 3028862622 ; E-mail: jeffrey.skolnik@ astrazeneca.com ${ }^{2}$ The Children's Hospital of Philadelphia, Clinical Pharmacology \& Therapeutics, Abramson Research Center, Room 916H, 3615 Civic Center Boulevard, PA 19104-4318, USA Tel.: +1 2155906359 Fax: +1 2155907544 E-mail:barrettj@ email.chop.edu

\section{'...despite these minor advances, pediatrics continues to use trial methodology first suggested over 60 years ago.'}

Despite recent advances in cancer pharmacology, the advent of targeted novel agents, and a greater understanding of dose-effect relationships, the trial design of pediatric Phase I studies has remained remarkably constant over the past 40 years. Recommendations from the National Cancer Institute over 10 years ago advocated the avoidance of traditional dose-escalation paradigms in favor of model-based approaches [1], yet significant modifications in study conduct have not been made. This is of concern because of extremely limited resources in access to both pediatric patients and to funding. Coupled with this is a lack of basic knowledge of the clinical pharmacology of most drugs used in children; over two-thirds of all drugs used in the pediatric population are used without proper labeling or pharmacokinetic information. Children are in an extremely vulnerable position because they are historically known to be at increased risk of toxicity from pharmacotherapy [2], and there are often no data that demonstrate efficacy for many of the drugs used for children. In 2002, the USA passed the Best Pharmaceuticals in Children Act (renewed in 2007), which was aimed at encouraging additional early-phase exploration for many agents used in pediatrics. Approximately 150 drugs now have additional dosing guidance thanks to this effort. However, despite these minor advances, pediatrics continues to use trial methodology first suggested over 60 years ago [3].

Recently, we published a novel clinical trial design for pediatric studies entitled the Rolling Six design [4]. The basics of this design are not fundamentally different than those that came before it; the overall goal of the Phase I study is to define the maximum tolerated dose (MTD) by assessing for toxicity through dose-limiting toxicity (DLT) events. In a traditional Phase I trial, patients are recruited in cohorts of three. Trials are suspended pending data return from each cohort of three patients before the trial can proceed. In the Rolling Six design, patients are recruited in cohorts of six, and trials are only suspended while waiting for data return on all six patients. This small change results in a 2-month reduction of study time, allowing MTDs to be defined quicker and Phase II studies to occur sooner. Our methodology will be validated prospectively as we adopt this design in current pediatric Phase I cancer trials. Although proposed for the pediatric cancer setting, this design would likely work in other fields of pediatrics; because much of the delay in completing Phase I trials comes from the time associated with patient accrual and inevaluability, and time to data submission and review, improvements in study performance, such as time-to-trial completion, lead to straightforward but necessary gains in conduct efficiency.

\section{'Future studies will need to depend more heavily on the use of biomarkers and biological and pharmacokinetic end points that define target inhibition.'}

Phase I trial designs will likely further evolve out of the need to accommodate new strategies of curative therapy that are moving away from nonspecific therapies and towards targeted approaches. Since classic Phase I methodology uses toxicity to define the MTD, agents that demonstrate a biological effect earlier than they demonstrate clinical toxicity may not be studied appropriately. In addition, many of the targeted agents rely on cytostasis rather than cytotoxicity, and conventional study end points may fail in these cases. Future studies will need to depend more heavily on the use of biomarkers and biological and pharmacokinetic end points that define target inhibition [5] in addition to DLT. A recent review of 60 single-agent Phase I oncology studies in adults demonstrated that in the majority of cases (36 out of 60) toxicity was still the primary reason for stopping dose escalation, and that in only $25 \%$ of cases (17 out of 60) were laboratory or radiographic studies used to inform dose selection [6]. Several adult Phase I investigators have favored alternative approaches to study design, including the Continuous Reassessment Method (CRM) [7], 
which attempts to predict toxicity, optimize dose-finding models and concentrate a higher percentage of patients enrolled to Phase I studies at or near the MTD. In the past, these methods have not shown a clinically relevant improvement in study conduct but would seem to offer great promise with respect to defining target doses [8]. Additional methodology will need to focus more on adaptive trial designs [9], pharmacokinetic-guided dosing [10] and pharmacogenomic-based dosing modification [11] in order to facilitate further improvement in both study outcome and efficiency.

'... one of the largest barriers to
early-phase pediatric studies has been
the unwillingness to involve children in
research largely deemed
'experimental' or 'exploratory'.'

Traditionally, investigators have been hesitant to subject children to agents for which there are limited safety data. However, pediatric clinical pharmacology benefits in having, in almost all cases, adult experiences prior to the start of most pediatric Phase I trials. This allows preliminary (adult) data on both the MTD and on the toxicity profile that can be brought forward into the associated pediatric study. In a recent review, Lee et al. demonstrated that for pediatric oncology Phase I trials, the pediatric MTD was rarely more than 1.6-times that of the adult MTD, and that these studies are safe [12]. By re-evaluating Phase I trial conduct in pediatric oncology, it was suggested that Phase I trials for these patients could be streamlined by starting studies at doses that are $70 \%$ of the known adult MTD, and by limiting the number of dose escalations to three. Similarly, one of the largest barriers to early-phase pediatric studies has been the unwillingness to involve children in research largely deemed 'experimental' or 'exploratory'. As Phase I agents are by definition investigational, both physicians and caregivers have been hesitant to involve pediatric patients in research that might not result in direct patient benefit [13]. More efficient and informative Phase I and II trials in children with cancer will certainly produce more specific guidance with respect to the management of individual patient pharmacotherapy. Continued improvements in trial design that optimize the limited resources available in pediatric clinical trials will be necessary in order to protect the safety of our patients, and to obtain the most scientifically helpful information in the most sensible way. The result of these changes will hopefully be more rapid access to new and exciting agents, with superior guidance on how we can optimally administer these therapies to our pediatric patients.

\section{Financial \& competing interests disclosure}

Jeffrey Skolnik is an employee of AstraZeneca, LP. The authors have no other relevant affiliations or financial involvement with any organization or entity with a financial interest in or financial conflict with the subject matter or materials discussed in the manuscript apart from those disclosed.

No writing assistance was utilized in the production of this manuscript.

\section{Bibliography}

1. Eisenhauer EA, O'Dwyer PJ, Christian M, Humphreys JS: Phase I clinical trial design in cancer drug development. J. Clin. Oncol. 18, 684-692 (2000).

2. Balis FM: Clinical Trials in Childhood Cancers. Oncologist 5, 2-3 (2000).

3. Reiner E, Paoletti X, O'Quigley J: Operating characteristics of the standard Phase I clinical trial design. Comput. Stat. Data Anal. 30, 303-315 (1999).

4. Skolnik JM, Barrett JS, Jayaraman B, Patel D, Adamson PC: Shortening the timeline of pediatric Phase 1 trials: the Rolling Six design. J. Clin. Oncol. 26, 190-195 (2008).

5. Fox E, Curt GA, Balis FM: Clinical trial design for target-based therapy. Oncologist 7 , 401-409 (2002).
6. Parulekar WR, Eisenhauer EA: Phase I trial design for solid tumor studies of targeted, non-cytotoxic agents: theory and practice. J. Natl Cancer Inst. 96, 990-997 (2004).

7. O'Quigley J, Pepe M, Fisher L: Continual reassessment method: a practical design for Phase I clinical trials in cancer. Biometrics 46, 33-48 (1990).

8. O'Quigley J, Zohar S: Experimental designs for Phase I and Phase I/II dosefinding studies. Br. J. Cancer 94, 609-613 (2006).

9. Normolle D, Lawrence T: Designing doseescalation trials with late-onset toxicities using the time-to-event continual reassessment method. J. Clin. Oncol. 24, 4426-4433 (2006).
10. Collins JM, Grieshaber CK, Chabner BA: Pharmacologically guided Phase I clinical trials based upon preclinical drug development, J. Natl Cancer Inst. 82, 1321-1326 (1990).

11. Maitland ML, DiRienzo A, Ratain MJ: Interpreting disparate responses to cancer therapy: the role of human population genetics. J. Clin. Oncol. 24, 2151-2157 (2006).

12. Lee DP, Skolnik JM, Adamson PC: Pediatric Phase I trials in oncology: An analysis of study conduct efficiency. J. Clin. Oncol. 33, 8431-8441 (2005).

13. Ross L: Phase I research and the meaning of direct benefit. J. Pediatr 149, S20-S24 (2006). 